\title{
A COMPARISON BETWEEN RANDOM AND DETERMINISTIC DYNAMICS OF RIVER DRAINAGE BASINS FORMATION
}

\begin{abstract}
C. A. Marin ${ }^{a}$, and M. R. Errerab

${ }^{\mathrm{a}}$ Universidade Federal do Paraná

Programa de Pós-Graduação em Engenharia de

Recursos Hídricos e Ambiental

Centro Politécnico

Bairro Jardim das Américas

CP. 19011, Curitiba, Paraná, 81540-350 Brasil

cesaugmarin@gmail.com

${ }^{\text {b} U n i v e r s i d a d e ~ F e d e r a l ~ d o ~ P a r a n a ́ ~}$ Departamento de Engenharia Ambiental

\section{ABSTRACT}

This work contributes to the discussion on how and why tree networks in drainage basin arises in nature. A fully deterministic erosion model recently developed was used to explain the spontaneous origin of river networks, which until then was assumed to be due to the optimal reduction of the flow resistance in a area to point flow. A random evolution model was devised in order to investigate the contribution of two separate features: the relative area size of the network and the structure (configuration) of the network. Two random models were applied, namely, restricted random choosing selection and fully random. Results showed that indeed the mere presence of low flow resistance portions of the basin does improve global performance and that randomness does not create networks. Further gain in performance is given by the network formation by erosion or by optimization. This suggest that local factors also are important to the explanation of the very existence of natural networks.
\end{abstract}

Centro Politécnico

Bairro Jardim das Américas

CP. 19011, Curitiba, Paraná, 81540-350 Brasil

errera@ufpr.br
Keywords: constructal theory, river drainage basins, tree networks, fractals

\section{NOMENCLATURE}

A surface area of the basin, $\mathrm{m}^{2}$

$\mathrm{D} \quad$ side of the square blocks, $\mathrm{m}$

$\mathrm{H} \quad$ width of the basin in $\mathrm{y}$-direction, $\mathrm{m}$

$\mathrm{K}$ permeability of the medium of the basin, $\mathrm{m}^{2}$

$\mathrm{Kp}$ permeability of the network path, $\mathrm{m}^{2}$

$\mathrm{L}$ length of the basin in $\mathrm{x}$-direction, $\mathrm{m}$

$\dot{\mathrm{m}}^{\prime \prime} \quad$ superficial mass flow rate, $\mathrm{kg} / \mathrm{m}^{2}$

$\mathrm{M}$ dimensionless mass flow rate, [-]

$\mathrm{N}$ number of eligible blocks, [-]

$\mathrm{n}$ evolution step, [-]

$\mathrm{P} \quad$ pressure or hydraulic potential, $\mathrm{Pa}$

$\mathrm{P}[\mathrm{]}$ probability of [...]

$\widetilde{\mathrm{P}}$ dimensionless hydraulic potential, [-]

$\widetilde{\mathrm{s}}$ dimensionless direction of resulting force over a block, [-]

$\mathrm{u} \quad$ velocity component at $\mathrm{x}$-direction, $\mathrm{m} / \mathrm{s}$

$\mathrm{v} \quad$ velocity component at $\mathrm{y}$-direction, $\mathrm{m} / \mathrm{s}$

$\mathrm{W}$ basin thickness in $\mathrm{z}$-direction, $\mathrm{m}$

$\mathrm{x}, \mathrm{y}, \mathrm{z}$ cartesian coordinates, $\mathrm{m}$

$\widetilde{\mathrm{x}}, \widetilde{\mathrm{y}}$ dimensionless cartesian coordinates, [-]

\section{Greek symbols}

$v \quad$ fluid kinematic viscosity, $\mathrm{m}^{2} / \mathrm{s}$

$\rho$ density, $\mathrm{kg} / \mathrm{m}^{3}$

$\tau$ maximum shear stress the basin matrix can withstand, $\mathrm{Pa}$
W identification number of a block, [-]

$\Omega \quad$ set of eligible blocks, [-]
Subscripts
$\mathrm{p} \quad$ related to high-permeability material
peak maximum pressure over the basin

\section{INTRODUCTION}

This paper is an expanded and up-dated edition of Marin and Errera (2009) that was kindly invited to be published in RET.

Tree networks are omnipresent forms in nature on a broad range of processes, e.g.: blood vessels configuration, nerves in leaves, dendritic solidification, roots distribution, bronchial trees, cracks in shrinking solids. Nature's resilience in maintain and make use of tree-like networks can be interpreted as a consequence of some global principle. The dynamic origin of these structures has been an important subject of study.

The features of river basins are widely known, mainly the morphology, that have been documented in various geophysics and hydrology treatises (e.g. Chorley et al (1984); Leopold et al (1964); Chow et al (1998) and Rodriguez-Iturbe \& Rinaldo (2001). One important feature documented is that river architectures exhibit similarities among a wide range of basins, computed on the form of scaling laws as 
the Horton's Laws, Melton's Law, and Hack's Law as pointed out in Leopold et al (1964). The fractal approach recently developed brought other scaling properties, which are based on statistical factors (Maritan et al, 1996). Hack's Law accounts for the elongation trend of basins, which is a regular criterion of evolution that has been interpreted as an evidence of randomness (Rodriguez-Iturbe \& Rinaldo, 2001).

The first models of river network formation were also devised in a completely random framework (Chorley et al (1984) and Cieplak et al, (1998). Afterwards it was shown that the assumption of equal likelihood of any tree-like configuration, inherent in these models, leads to the creation of networks that do not reproduce well the scaling characteristics verified in real river basins (Rinaldo et al, 1998). When the concept of minimum energy expenditure is used, the random models were adapted to a concept now known as Optimal Channel Networks (OCN). The OCNs are created in an algorithm that starts with a random network, and make random changes channel by channel in the structure - a similar principle as Darwin's Natural Selection for organisms traits. The changes are accepted only if the new configuration reduces the value of a functional related to the energy expenditure (Rinaldo et al, 1998 and Rinaldo et al, 2006). The configurations obtained with this method are able to reproduce well the scaling laws parameters found in nature.

Nevertheless one important challenge remains unsolved after these achievements. The OCN creation uses a physical concept, but starts with the prior assumption that the networks exists - this very assumption, though, does not comply with Darwin's theory that is the basis of the OCN method. In other words, it fails to explain why nature "chooses" to use a network instead of another geometrical form of drainage basin (e.g. a lake or a pond, or a single wider channel). There are also deterministic models of fluvial landform evolution (e.g. Howard, 1997 and Pelletier, 2007) that consider the effects of mass diffusion, erosion, and tectonic uplift in the shape of the landforms, but the networks only appear because of the initial conditions or because of random erodibility (Pelletier, 2007).

The origin of networks in river basins was approached in Errera and Bejan (1998), where concepts of Constructal Theory (CT) were firstly applied on the subject. Constructal Law was first presented in Bejan (1997a) as follows: "For a finitesize system to persist in time (to live), it must evolve in such way that it provides easier (and easier) access to the imposed (global) currents that flow through it". It deals with the generation of flow configuration in nature in a deterministic manner (Bejan, 1997b), mainly with the global characteristics that are not driven by local restraints (Bejan and Tondeur, 1998).

Bejan and Lorente (2006) and Bejan (2007) are recent reviews that show a wide array of configurations of natural phenomena that can be predicted by this theory in both inanimate systems (e.g. duct cross sections, open channel cross sections, turbulent flow structure, global circulation and climate) and animate systems (e.g. allometric laws of body size, breathing, flying running and swimming), along with applications in engineering (e.g. heat conduction, heat exchangers, fluid flow). Therefore it can be seen that CT has successfully demonstrated the why of the very existence of the various types of flow configurations in nature, and it did it in a completely deterministic approach.

Eventually one will be able to obtain, as a clear and plain result, very similar forms of existing river basins by straight-forward application of CT.

Tree networks, as a variation form of drainage basin, was firstly approached by $\mathrm{CT}$ with a analytical formulation based on several simplifying assumptions: e.g. right angles between stems and tributaries, fully developed laminar flow, a chosen construction sequence, constant-thickness branches (Bejan, 1997a, Bejan, 1997c and Bejan and Errera, 1997). Subsequently, most of the assumptions were relaxed, and the same problem was solved numerically (Ledezma et al, 1997 and Errera and Bejan, 2000). The networks in those works resulted from the need for connecting an infinite amount of points (e.g. a volume, a area or a line) to a single point (a source or a sink), balancing two flow schemes, to create the least global resistance possible (Bejan and Lorente, 2006).

In the river basins case, the higher resistance flow takes place in porous medium, and the lower resistance flow is the open channel flow (the network). Recently, it was shown that the principal scaling laws of river networks can be deterministically deduced, including the tendency of elongation reported through Hack's Law (Reis, 2006). It was used an analytical treatment similar to Bejan (1997a) applied to river networks (Bejan, 1997b). In this case, the ordering scheme was still assumed.

On the other hand, in Errera and Bejan (1998) the network appears spontaneously based only on an erosion model, without any assumption about ordering scheme or initial conditions. That is, the basin self-constructed its network. It is shown that this process leads to a reduction of the global resistance. Such reduction takes place firstly because the placing of lower flow resistance paths on the space previously occupied by higher flow resistance is a process that reduces the global resistance per se. Later a second reduction comes to play because of "which" space is converted to low resistance.

Errera and Bejan (1998) also tested evolution by optimization (minimization of global flow resistance) at every step of change. They showed that configurations created in the erosion model was very close to the optimum. That is, an optimization 
approach is unnecessary to produce networks or not present at all in nature.

This work therefore investigated the earlier mentioned second contribution of the erosion process, that is, the differences in global flow reduction given by "which" space is converted to low flow resistance path.

The simplest way to check is to compare the global performance of a structure evolved by just randomly replacing parts of the original basin to the evolution by the erosion model of Errera and Bejan (1998) for the same amount of area turned into low flow resistance path.

By doing so, a secondary question is also answered: will random replacing (evolution) produce configurations that resembles tree flow networks ?

Purely (fully) random evolution is the extreme of the approaches earlier presented in Chorley et al (1984), Cieplak et al (1998), Rinaldo et al (1998) and Rinaldo et al (2006).

The results show that the global performance of the random configurations follow the same trend of decrease of global resistance of the erosion configurations, but they are slightly poorer than the last ones. The patterns created are similar to diffusion process, with the same order of magnitude of the global performances.

\section{SIMULATION METHODS}

The erosion model of Errera and Bejan (1998), which is fully deterministic, was adapted to reproduce a fully random dynamics. The original model is briefly represented in Fig. 1. A basin of a surface area of $\mathrm{A}=\mathrm{HL}$ and shape $\mathrm{H} / \mathrm{L}$ fixed is initially coated with a homogeneous porous layer of permeability $\mathrm{K}$, with a very small thickness $\mathrm{W}<<$ $(\mathrm{H}, \mathrm{L})$.

The area A receives a uniform mass flow rate of an incompressible Newtonian fluid (in the case of water, this is similar to rainfall) of value $\dot{m}^{\prime \prime}$, that is fully discharged through a small opening of size D $x$ $\mathrm{W}$ placed over the origin of the system. The load thus creates a pressure field (hydraulic potential) $\mathrm{P}(\mathrm{x}, \mathrm{y})$ that drives the fluid to the outlet. The flow through the $\mathrm{K}$ medium is modeled as Darcy flow. Hence the pressure field can be obtained by a Poisson type equation:

$$
\frac{\partial^{2} P}{\partial x^{2}}+\frac{\partial^{2} P}{\partial y^{2}}+\frac{\dot{m}^{\prime \prime} v}{W K}=0
$$

where $v$ is the kinematic viscosity of the fluid.

Spaces with open channel flow can be created because the soil blocks (DxD) can be dislodged (Fig.1). This happens when the force created by the pressure field acting on the block $(\triangle \mathrm{PDW})$ is stronger than the cohesion force of the soil $\left(\tau \mathrm{D}^{2}\right.$, where $\tau$ is the maximum shear stress supported by a block). On these terms, Eq. (1) is changed to a dimensionless form:

$$
\frac{\partial^{2} \widetilde{P}}{\partial \widetilde{x}^{2}}+\frac{\partial^{2} \widetilde{P}}{\partial \widetilde{y}^{2}}+M=0
$$

where:
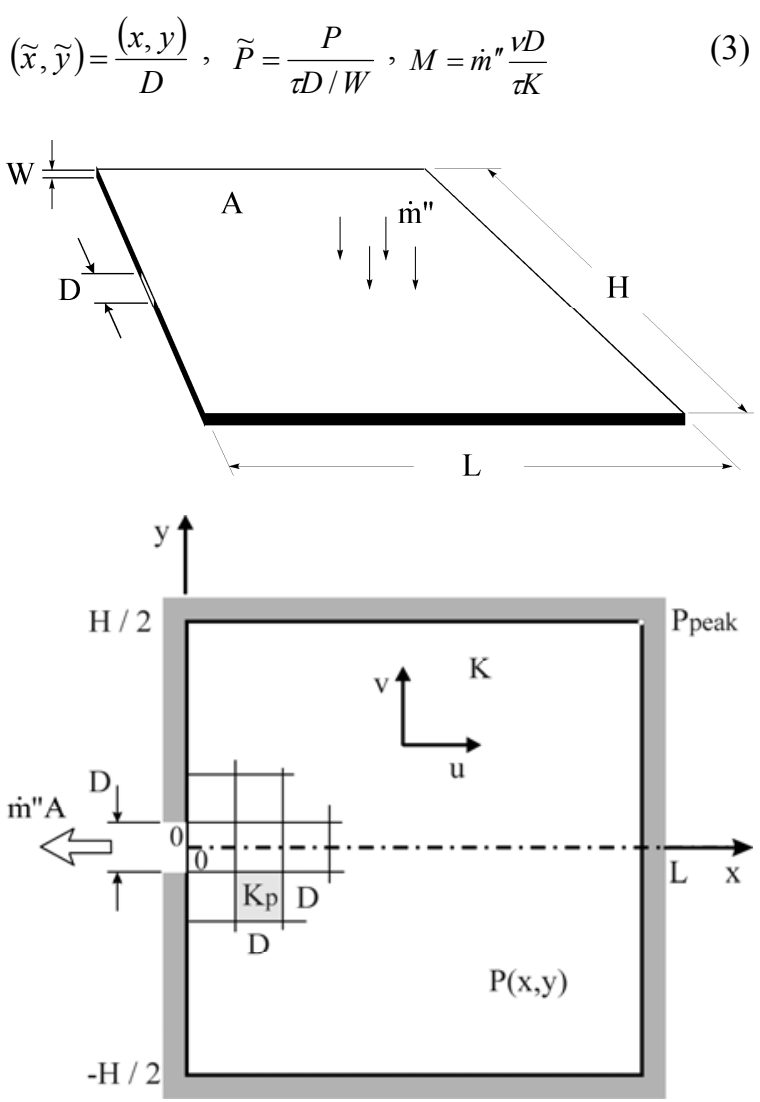

Figure 1. Two-dimensional model of an area-to-point flow in a porous medium with the soil simulated as square blocks of side $\mathrm{D}$, that can be dislodged to form an open channel flow (Errera and Bejan, 1998)

In this case, if $s$ is the direction of the resulting force acting on the block, the block is not dislodged as long as:

$$
\left(\frac{\partial \widetilde{P}}{\partial \widetilde{S}}\right)<1
$$

which gives the condition to the removal of the blocks.

In every step of the simulation, this condition was applied to the blocks that had one of the sides facing to a previous removed block, which are called eligible blocks. The first block to be removed is the one facing to the outlet, and the dynamics follows from there. The place from where blocks were removed become a new domain of "open channel", that it was assumed to be also a Darcy flow, but with a significant less flow resistance, translated in a higher permeability Kp. Equation (1) is adapted to the Kp-medium by multiplying $\mathrm{M}$ by the ratio $\mathrm{K} / \mathrm{Kp}$. 
Errera and Bejan (1998) used the condition of Eq. (4) on the evolution dynamics so the removed blocks were chosen without interference. In this work that condition is not applied, that is, the mechanics of the evolution is relaxed.

Instead of using the physics of the erosion problem, or an optimization procedure, the eligible blocks now have equal likelihood of being removed. They are randomly dislodged based on series of uniform distribution created for every step of the dynamics. From a mathematical viewpoint, the removal criterion is based on the following relationship:

$$
P[\Omega=\omega]=\frac{1}{N_{\Omega}}
$$

where $\omega$ is the identification number of block to be removed, $\Omega$ is the set of all the available blocks at a step $n$, and $\mathrm{N}_{\Omega}$ is the size of this set. At this point, it must be clear that $\Omega$ is a discrete set of random variables. This model will be often referred as "random choosing model".

After that, the deterministic feature of the evolution dynamics holds only to determine of the set of eligible blocks (adjacent of a Kp region).

Symmetry was assumed with respect to the $x$ axis solely for computational performance.

In a second simulation the randomness of the dynamics was further increased by allowing any Ktype block to be selected for dislodging (to become $\mathrm{Kp}$ ). Once again, blocks have equal likelihood of being removed. Now there are two sets of random block selection, namely, restricted and fully.

In Errera and Bejan (1998), it was shown that local differences are created due to the sequence in which the value of $M$ is increased (though the global resistance is almost insensitive to this). In the present work the configuration does not depend of physical factors, hence it is not affected by the values of $\mathrm{M}$.

The ultimate objective of the current analysis is to compare the configurations obtained in the present work (random) to the ones obtained using the erosion model (physical) and the optimization procedure. Among the simulations carried out by Errera and Bejan (1998), it was chosen two for the comparison: erosion model considering $\Delta \mathrm{M}=0.001$ and the optimization evolution model.

In both cases were set $\mathrm{H}=\mathrm{L}=51 \mathrm{D}$, which in turn leads to a first critical value $\mathrm{M}_{\mathrm{c}}$ of 0.00088932 to the erosion model (the value to the removal of the first block).

The flow resistance is measured as the ratio between the peak pressure (highest pressure across the domain) and the flow forcing (M) term, namely:

$$
\text { Flow Resistance } \propto \frac{\max (\widetilde{P})}{M}=\frac{\widetilde{P}_{p e a k}}{M}
$$

This ratio is used to compare the gains achieved among evolution processes considered.

The model and the computer code was up-dated for today's computational capabilities and versions of compilers and FIDAP ${ }^{\circ}$. The new code was successfully validated by comparing the counterpart of Fig. 2a and Fig. 2b of Errera and Bejan (1998).

\section{RESULTS AND DISCUSSION}

A set of six simulations with the restricted random condition set in Eq. (5) were carried out. They are shown overlapped in Fig. 2c for the sake of conciseness.

To read the patterns in Fig. 2c one shall consider the degree of shading, which is associated to the observed times in which a block was selected until a given step $n$ : the darkest (black) blocks were chosen six times (in all simulations), the lightest (white as the background) none.

One can notice dramatic differences in the configurations generated by Fig. 2a (mechanical erosion) and Fig. 2c (restricted randomness). The statistical expectancy of the structure derived by random dynamics (overlap) resembles a "diffusive" process of the Kp domain that was also predicted by Errera and Bejan (1998) (Fig. 5), when the flow resistances of $\mathrm{K}$ and $\mathrm{Kp}$ media was almost the same, i.e., $\mathrm{K} / \mathrm{Kp} \sim 1$.

In other words, the restricted (choosing) random selection by Eq. (5) of eligible blocks (those adjacent to a Kp block) may well represent the case of erosion in which there is no gain in forming preferential paths. Furthermore results also show that no network pattern will be formed if it is not assumed à priori as it was done in Chorley et at (1984) and Cieplak et al (1998).

Even though networks are not formed in the restricted random simulation, "fingers" may appear. Most of them appear and later vanish. It is reasonably to state that such occurrences are not based on physical laws.

Figure $2 \mathrm{~d}$ shows that in the fully random model no networks, neither any structure is identified.

Another important feature is that all the configurations in Fig. 2 clearly show that networks, that are complex geometrical forms, can be derived from simpler ones, as the diffusion patterns.

The flow resistances generated by the configurations of Fig. 2 are plotted against the number of dislodged blocks in Fig. 3, considering the ratio $\mathrm{K} / \mathrm{Kp}=0.1$ (each flow field was calculated). That shows the trend of flow resistance to decrease in either erosion and random models. In the latter the gain with the removal of one block is almost the same along the dynamic steps.

In the early steps of both erosion model and random choosing model the gain for just replacing the original blocks for high permeable blocks provided significant decrease in the global flow 
resistance. In fact they are near the same up to step $n$ $=50$. The fully random, on the other hand, show improvements but performs relatively poorly, nearly the double of the erosion model.

The best performance is given by the optimized selection model (Fig. 2b), based on which a new comparison can be made. In Figure 4, we show the relative gain in the global flow resistance of each model (average performance random models).

a
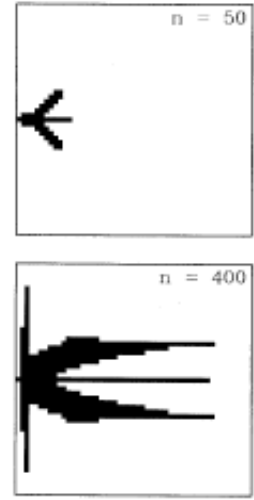

b
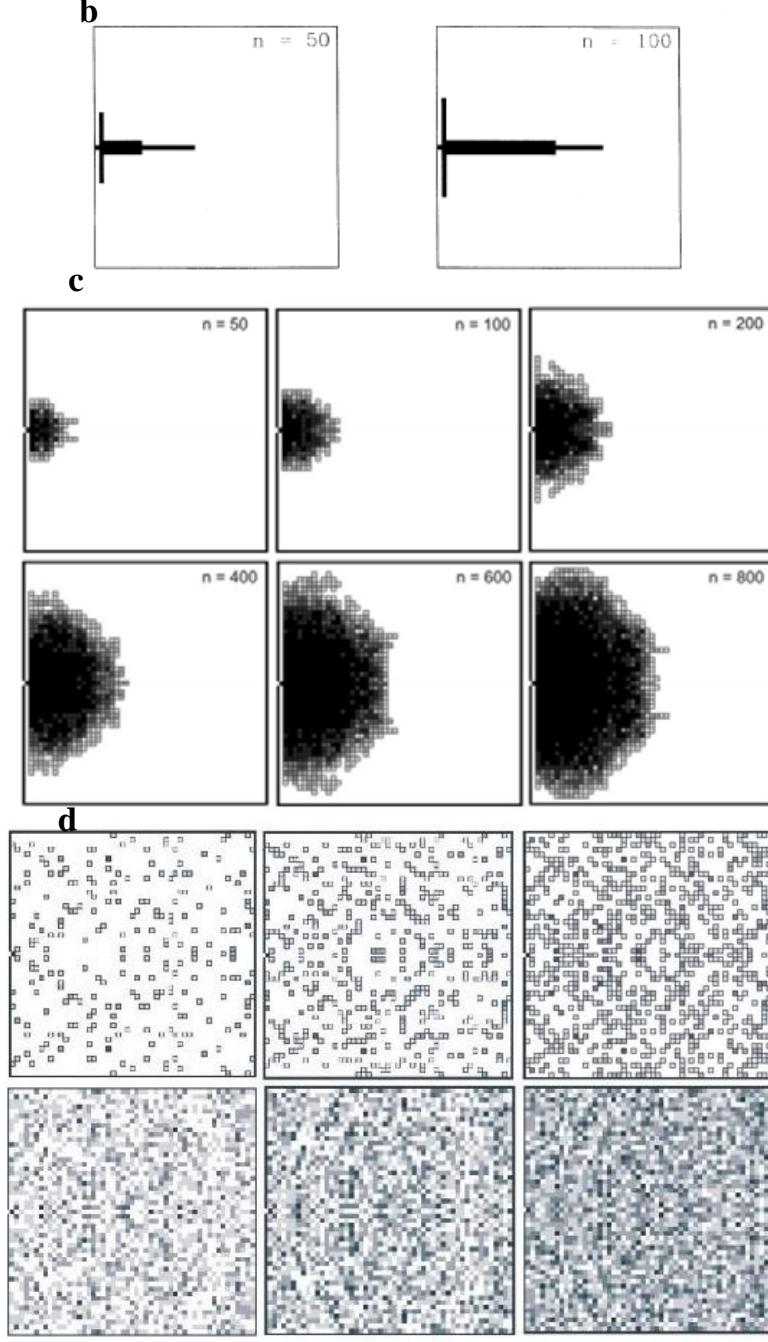

Figure 2. Evolution of the basin for $\mathrm{K} / \mathrm{Kp}=0.1$ and $\Delta \mathrm{M}=10^{-3}$; a) Erosion; b) optimization model; average of 6 evolutions random choosing model c) and d) fully random model, (black means six times and white means none).

The plot in Fig, 4 shows clearly that the reduction in the global flow resistance due to introduction (replacement) of low permeable to high permeable material is not enough to explain whole gains in performance: the self-constructed flow network of the erosion model performs significantly better, up to half of the resistance.

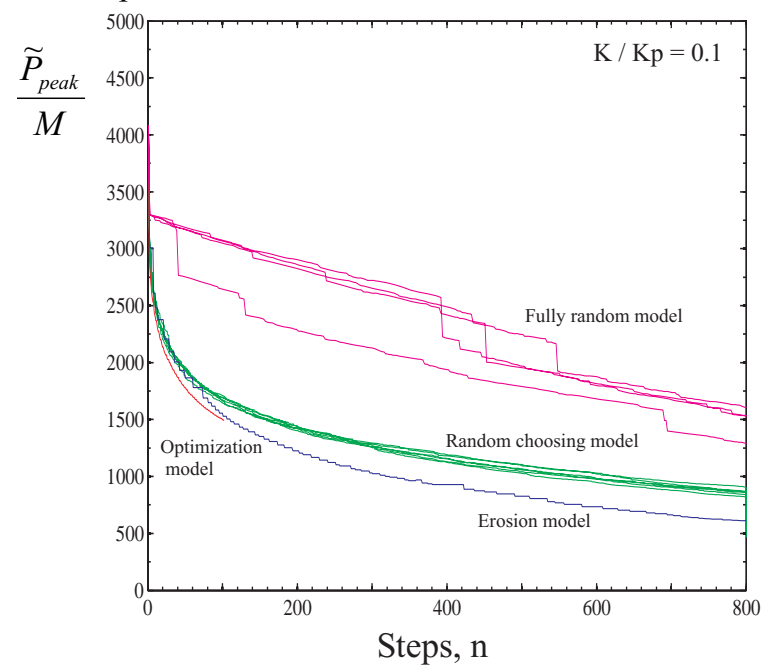

Figure 3. Variation of global flow resistance in the evolution process of the erosion model, the restricted random model and the fully random model.

It is worth mentioning that, in nature, even slight differences in performance has shown to be decisive in selecting forms, shapes and structures.

Another way to look in those dynamics is to consider that in the random choosing method, the degree of freedom is smaller than the fully random and yet it performs better. This loss of freedom was not arbitrary, it was associated with gain of information.

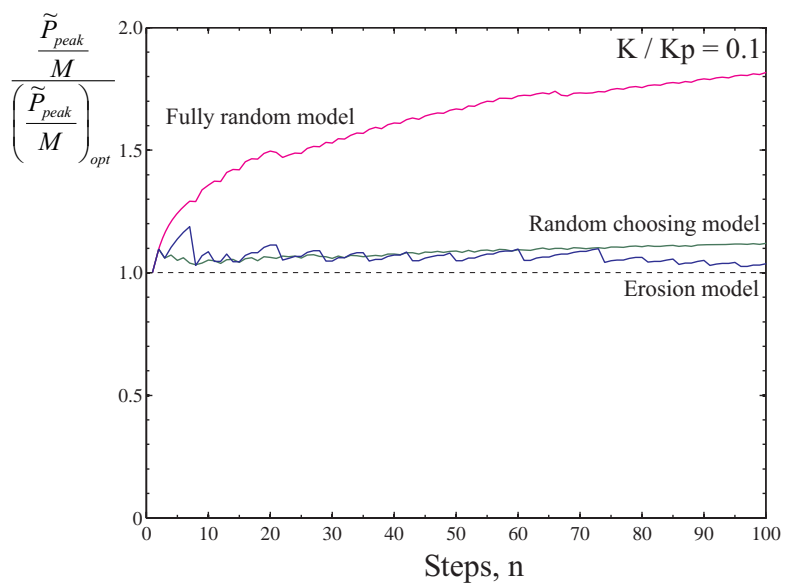

Figure 4. Early stages of the ratio between the flow resistance obtained in the erosion and average of random models and the ones obtained with the optimization models, for $\mathrm{K} / \mathrm{Kp}=0.1$. The dashed line represents the unity. 
When one considers the fact the random choosing model follows the erosion and optimum models (that are deterministic) very closely in the early stages in terms of the global performance (Figs. 3 and 4), one may ask that global performance might not be the only feature in the network creation.

Based on the results of Fig. 4, it cannot be said that the network was created only because of the need of the global flow resistance reduction. It must have at least one more factor that is important to the dynamics, which can be the need of local performance gain, as represented by the erosion model. That is, the changing of portions subjected to high local pressures.

It is important to see in Fig. 3 that the dispersal of the monotonic trend of resistance reduction created by the random simulations are almost the same. This shows that the number of six random simulations done in the present work was good enough for the goals set.

\section{CONCLUSIONS}

In this paper we determined the contribution of the erosion model to explain the dynamics of formation of networks in a idealized drainage basin. The contribution of solely replacing low permeability material by higher permeability material is limited, showing that indeed the self-constructed flow network further reduce the global flow resistance.

The results also indicate that the global performance is significant in the shaping of these forms, but does not fully explain all the phenomenon.

The degrees of freedom play a special role in this matter. The networks are only formed when, in any step, there is only one block that can be removed, that is the most "stressed" one (these are the deterministic models). In the random choosing case, this number gets higher at every step and networks are not created.

Finally it is important to realize that the results obtained here, together with the ones of Bejan and Errera (1998), may a be proof that complex structures like the networks can be derived from simpler ones, in fact the simplest. In particular, this shows that the non-scientific doctrine of the "irreducible complexity" is very inconsistent in the river networks example, since it claims that networks can only be consequence of other smaller networks. The results obtained here are a clear proof that this is a inconsistent argument.

New questions arose and new studies will be carried out to help fill the blanks Constructal Theory has yet to face.

\section{ACKNOWLEDGEMENTS}

We wish to acknowledge Coordenação de Aperfeiçamento de Pessoal de Ensino Superior
(CAPES) for the educational and financial support and Duke University for the cooperation on the use of the FIDAP ${ }^{\circledR}$ software and the its computers. We also would like to thank Prof. Luiz Rocha's support.

\section{REFERENCES}

Bejan, A., 1997a, Constructal-theory network of conducting paths for cooling a heat generating volume, Int. J. Heat Mass Transfer, Vol. 40, pp 799816.

Bejan, A., 1997b, Advanced Engineering Thermodynamics, 2nd Ed, New York, USA: Wiley.

Bejan, A., 1997c, Constructal tree network for fluid flow between a finite-size volume and one source sink, Rev. Gén. Therm., Vol. 36, pp. 592-604.

Bejan, A., 2007, Constructal theory of pattern formation, Hydrol. Earth Syst. Sci., Vol. 11, pp 753768.

Bejan, A., and Tondeur, D., 1998, Equipartition, optimal allocation, and the constructal approach to predicting organization in nature, Rev. Gén. Therm., Vol. 37, pp. 165-180, 1998.

Bejan, A., and Errera, M.R., 1997, Deterministic tree networks for fluid flow: geometry for minimal flow resistance between a volume and one point, Fractals, Vol. 5, pp. 685-695.

Bejan, A., S. and Lorente, 2006, Constructal theory of generation of configuration in nature and engineering, J. Appl. Phys., Vol. 100, pp. 1-27.

Chorley, R. J., Schumm, S. A. and Sugden, D. E., 1984, Geomorphology, Ed. New York, USA: Methuen \& Co.

Chow, V. T. , Maidment, D. R. and Mays, L. W., 1998, Applied Hydrology, Ed. New York, USA: McGraw-Hill Inc.

Cieplak, M., Giacometti, A., Maritan, A., Rinaldo, A., Rodriguez-Iturbe, I. and Banavar, J. R., 1998, Models of fractal river basins, Journal of Statistical Physics, Vol. 91, pp. 1-14.

Errera, M. R., and Bejan, A., 1998, Deterministic tree networks for river drainage basins, Fractals, Vol. 6, pp. 245-261.

Errera, M. R., and Bejan, A., 2000, Tree networks for minimal flow resistance to volume-topoint flows in composite porous media, Journal of Porous Media, Vol. 2, pp.1-18.

Howard, A. D., 1997, Badland morphology and evolution: interpretation using a simulation model, Earth Surf. Processes, Vol. 22, pp 211-227.

Ledezma, G. A., Bejan, A. and Errera, M. R., 1997, Constructal tree networks for heat transfer, J. Appl. Phys., Vol. 82, pp 89-100.

Leopold, L. B., Wolman, M. G. and Miller, J. P., 1964, Fluvial Processes in Geomorphology, Ed. San Francisco, USA.

Marin, C. A. and Errera, M. R., 2009, A comparison between random and deterministic tree networks for river drainage basins, Third Southern 
Conference on Computational Modeling (3MCSUL), IEE Proceedings (2010), pp.18-23

Maritan, A., Rinaldo, A., Rigon, R., Giacometti, A. and Rodriguez-Iturbe, I., 1996, Scaling laws for river networks, Physical Reviews E, Vol. 53, pp. 1510-1515, 1996.

Pelletier, J. D., 2007, Fractal behavior in space and time in a simplified model of fluvial landform evolution, Geomorphology, Vol. 91, pp 291-301.

Reis, A. H., 2006, Constructal view of scaling laws of river basins, Geomorphology, Vol. 78, pp. 201-206.

Rinaldo, A., Banavar, J. R. and Maritan, A., 2006, Trees, networks, and hydrology, Water Resour. Res., Vol. 42, pp. 1-19.

Rinaldo, A., Rodriguez-Iturbe, I. and Rigon, R., 1998, Channel networks, Annu. Rev. Earth Planet. Sci., Vol. 26, pp. 289-327.

Rodriguez-Iturbe, I. and Rinaldo, A. 2001, Fractal River Basins, Ed. Cambridge, UK: Cambridge University Press.

Received: September 04, 2008

Revised: October 04, 2008

Accepted: November 04, 2008 